Supporting Information for

\title{
Aromatic Fragmentation Based on a Ring Overlap Scheme: An Algorithm for Large Polycyclic Aromatic Hydrocarbons Using the Molecules-in-Molecules Fragmentation-Based Method
}

\author{
Benjamin W. Noffke, ${ }^{\dagger}$ Daniel Beckett ${ }^{\dagger}$ Liang-shi Li, and Krishnan Raghavachari* \\ Department of Chemistry, Indiana University, Bloomington, IN 47405
}

\section{Table of Contents}

1. AroBOROS Acronym Explanation $\quad$ S2

2. Ethene Cancellation Example $\quad$ S2

3. Exploded Diagrams of Test Molecules’ Fragment Sets S3

${ }^{\dagger}$ B.W.N. and D.B. contributed equally to this work.

* Corresponding author: kraghava@indiana.edu 


\section{AroBOROS Acronym Explanation}

The acronym is meant to be a play on the Greek word "ouroboros": a snake/dragon drawn biting its own tail. Kekule proposed the correct structure of benzene after dreaming of an ouroboros. Additionally, the ouroboros has long been a symbol in alchemy, making it a culturally relevant picture for chemistry. In Norse Mythology, there is an ouroboros that initiates the end of the world by releasing its tail.

One can correlate the ideal Clar structures of a PAH to embedded benzene rings (aromatic sextets), each representing an ouroboros. The whole view of the PAH reveals several ouroboroi. The AroBOROS method avoids disrupting rings of aromatic sextets, analogously stopping an ouroboros from releasing its tail and causing the end of the world.

\section{Ethene Cancellation Example}

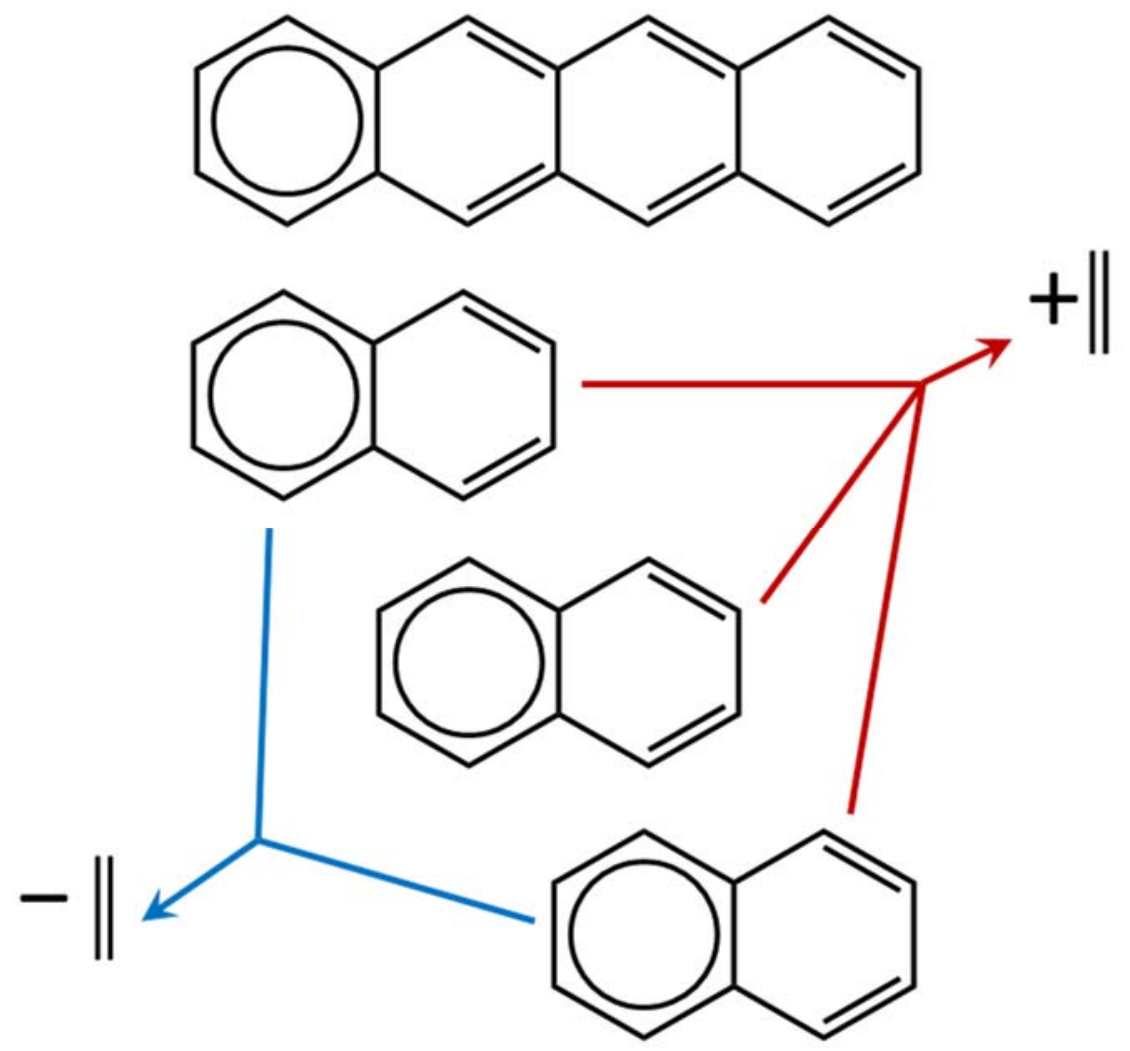

Figure S1. Fragment set for tetracene showing the ethene cancellation in the two- and three-body overlaps. 
The main text briefly discusses the tracking of ethene derivative subsystems in two- and three-body overlaps for potential cancellation. This is required because the full inclusion-exclusion principle (IEP) is not applied in the algorithm to determine the fragment set. Figure S1 shows an example of how this cancellation can manifest itself in tetracene. Such cancellation will be present in almost any system that requires a naphthalene in its fragment set. The full IEP is then applied after the fragment set is determined, in which case the cancellations will explicitly happen and only whole ring derivative subsystems will be present.

\section{Exploded Diagrams of Test Molecules' Fragment Sets}

The following figures illustrate the composition of subsystems in the fragment set for each test system at Rungs 1 and 2. The exploded fragment diagram for triphenylene, tetracene, and CCC48 (kekulene) can be found in Figures 3, 4, and 7, respectively. 


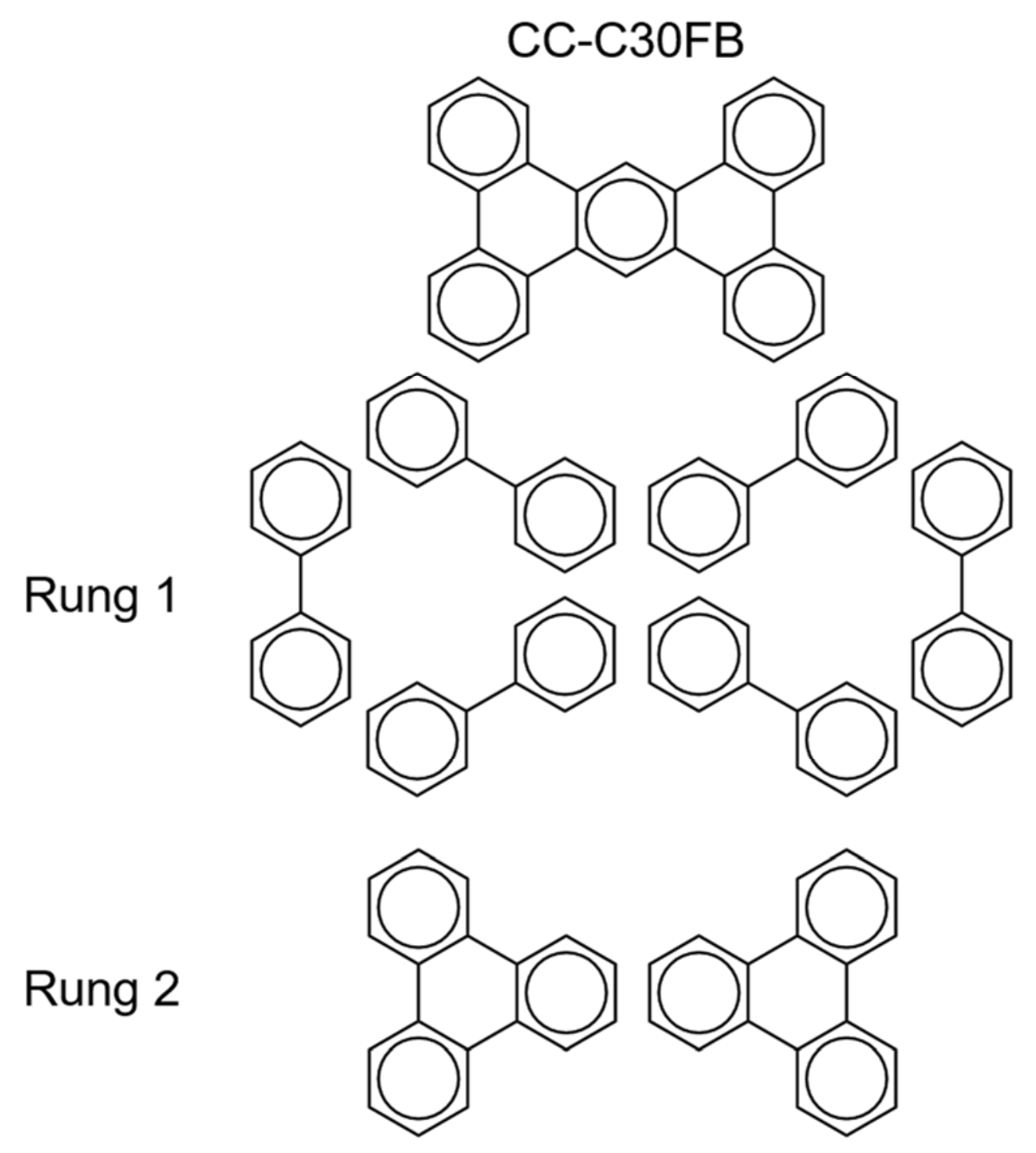




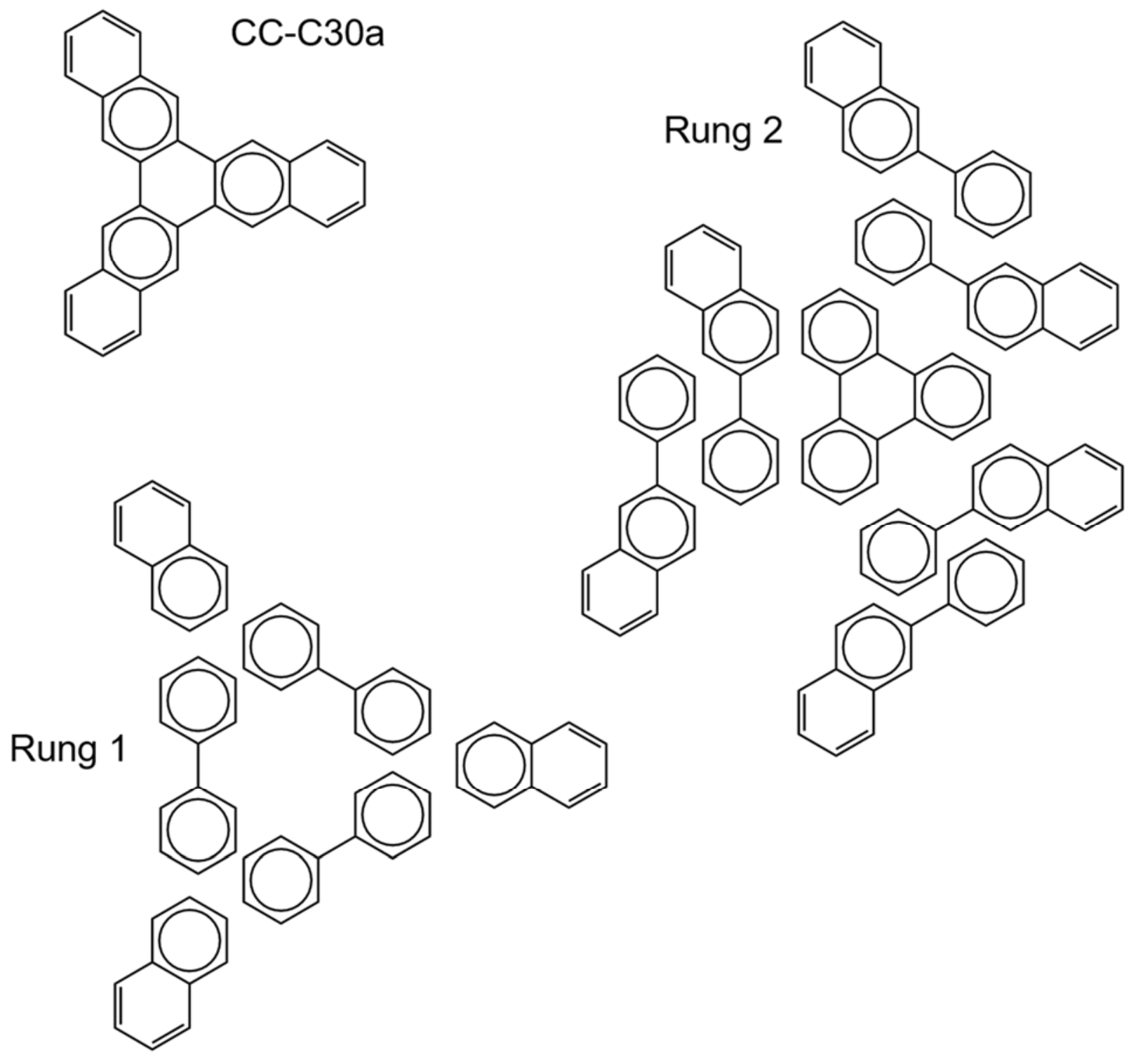




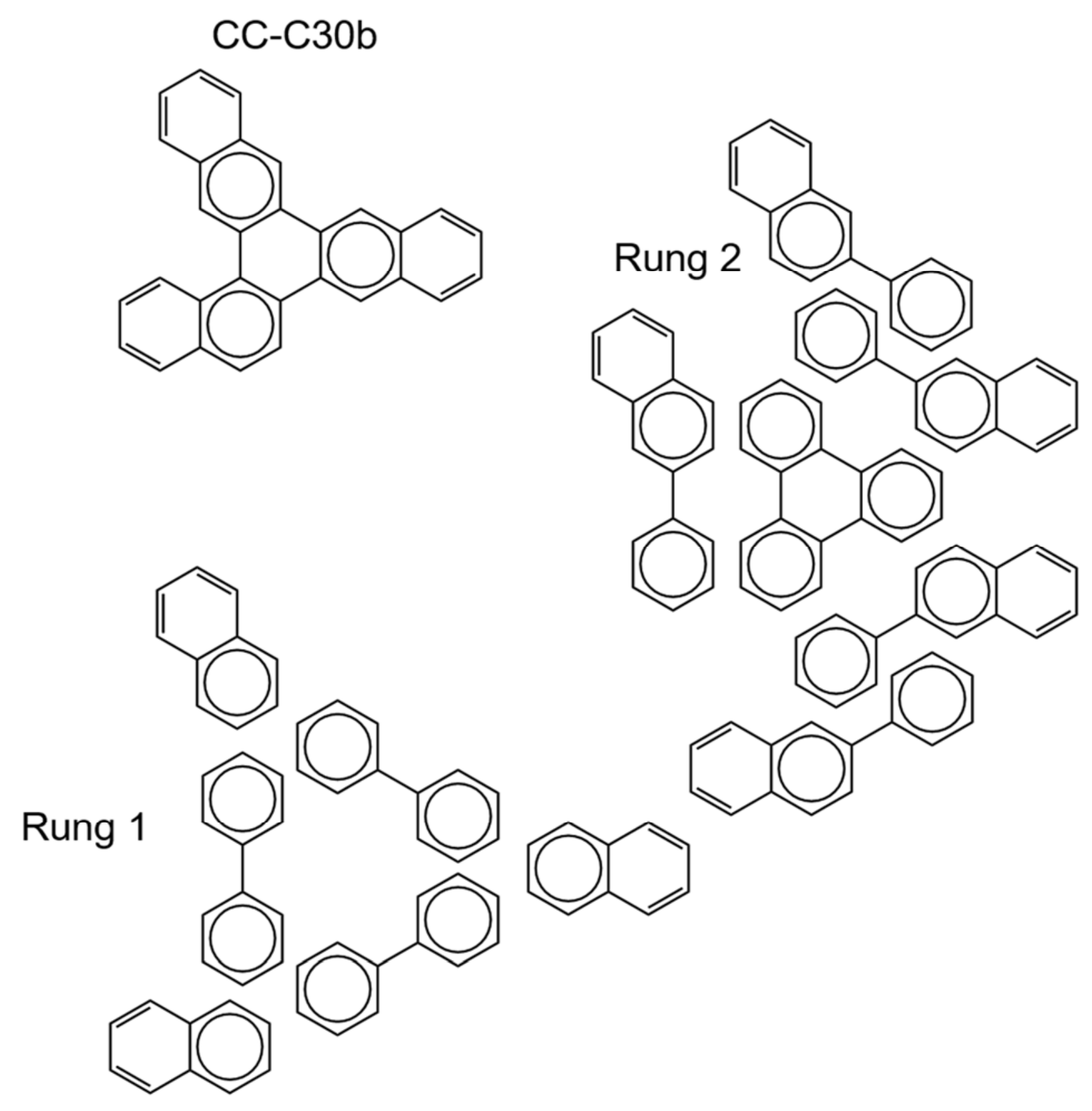




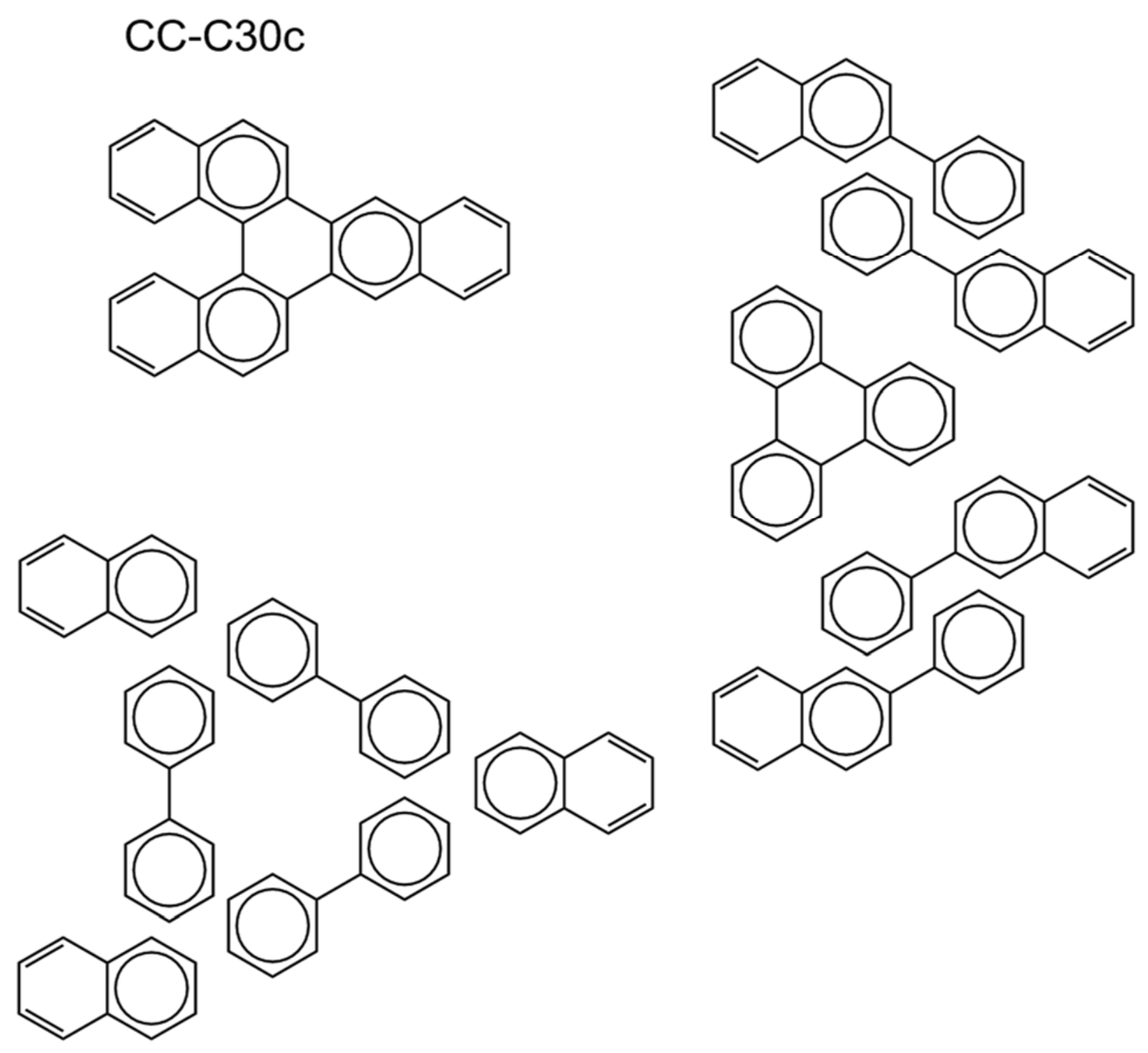




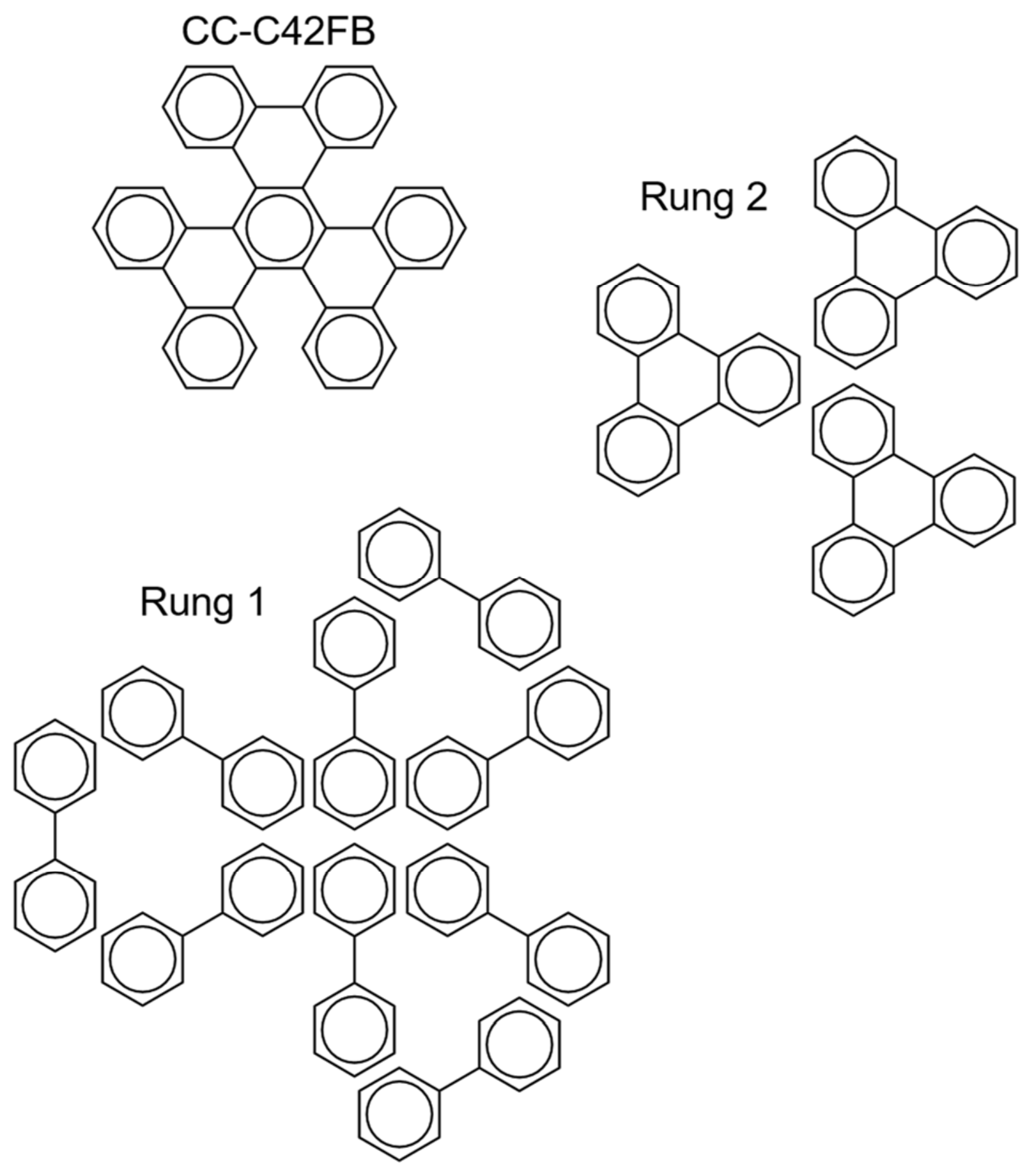




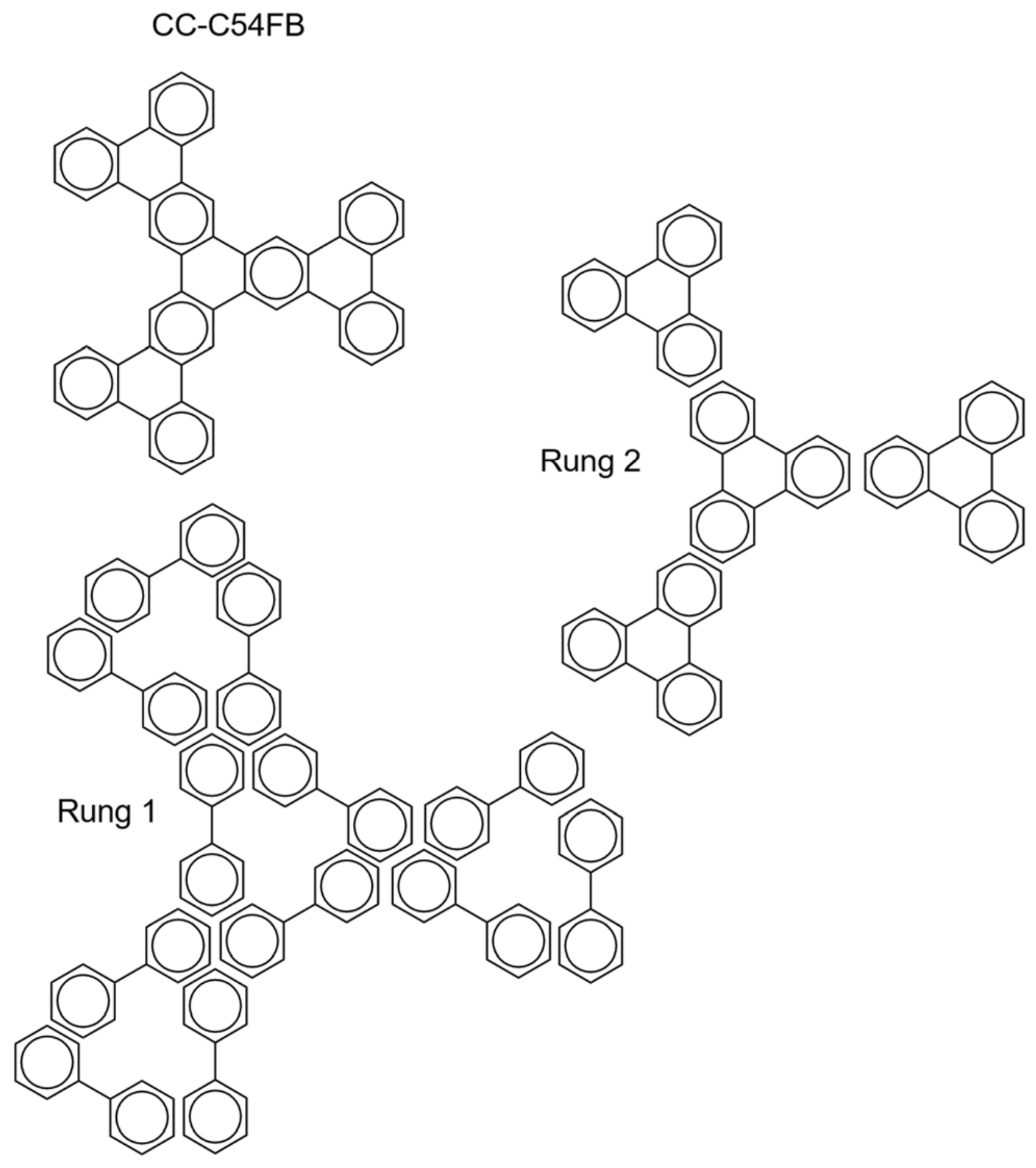




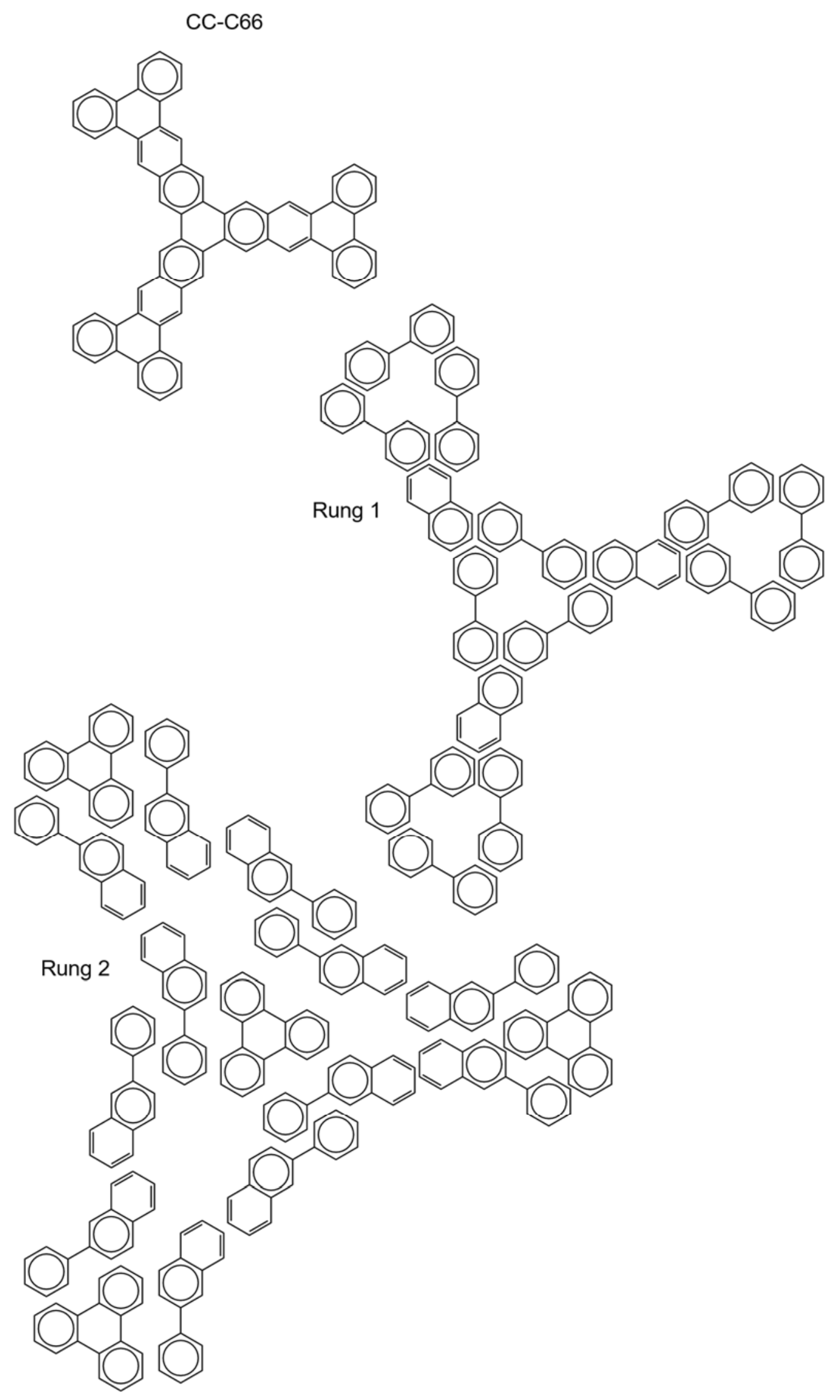



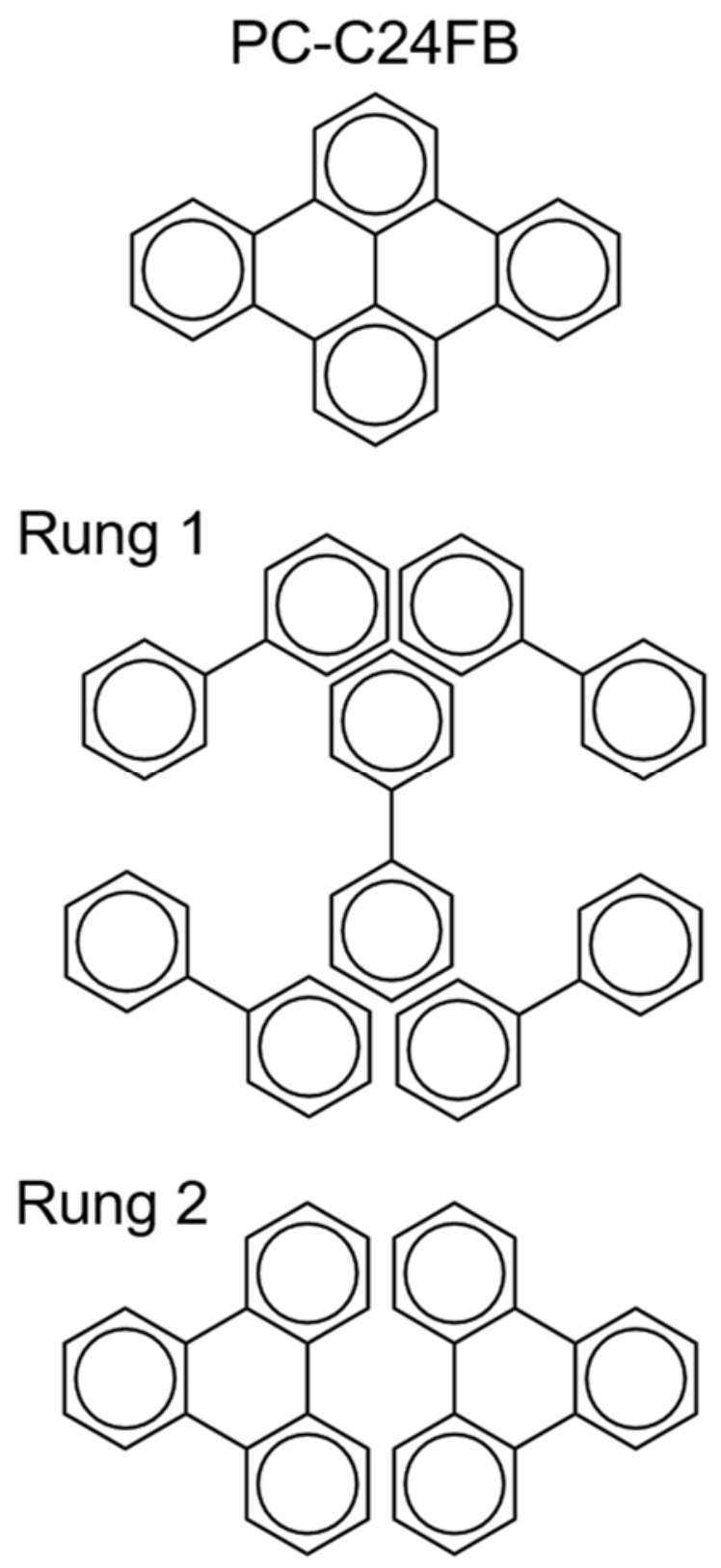

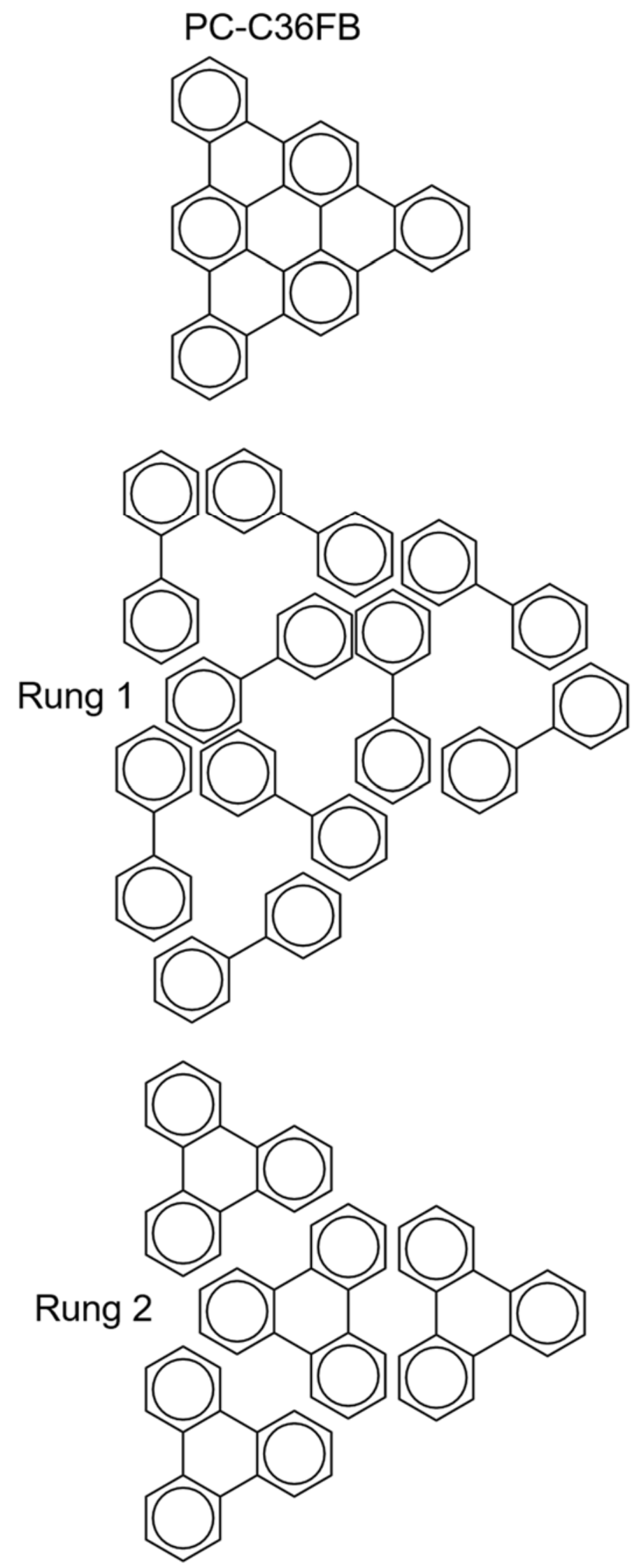


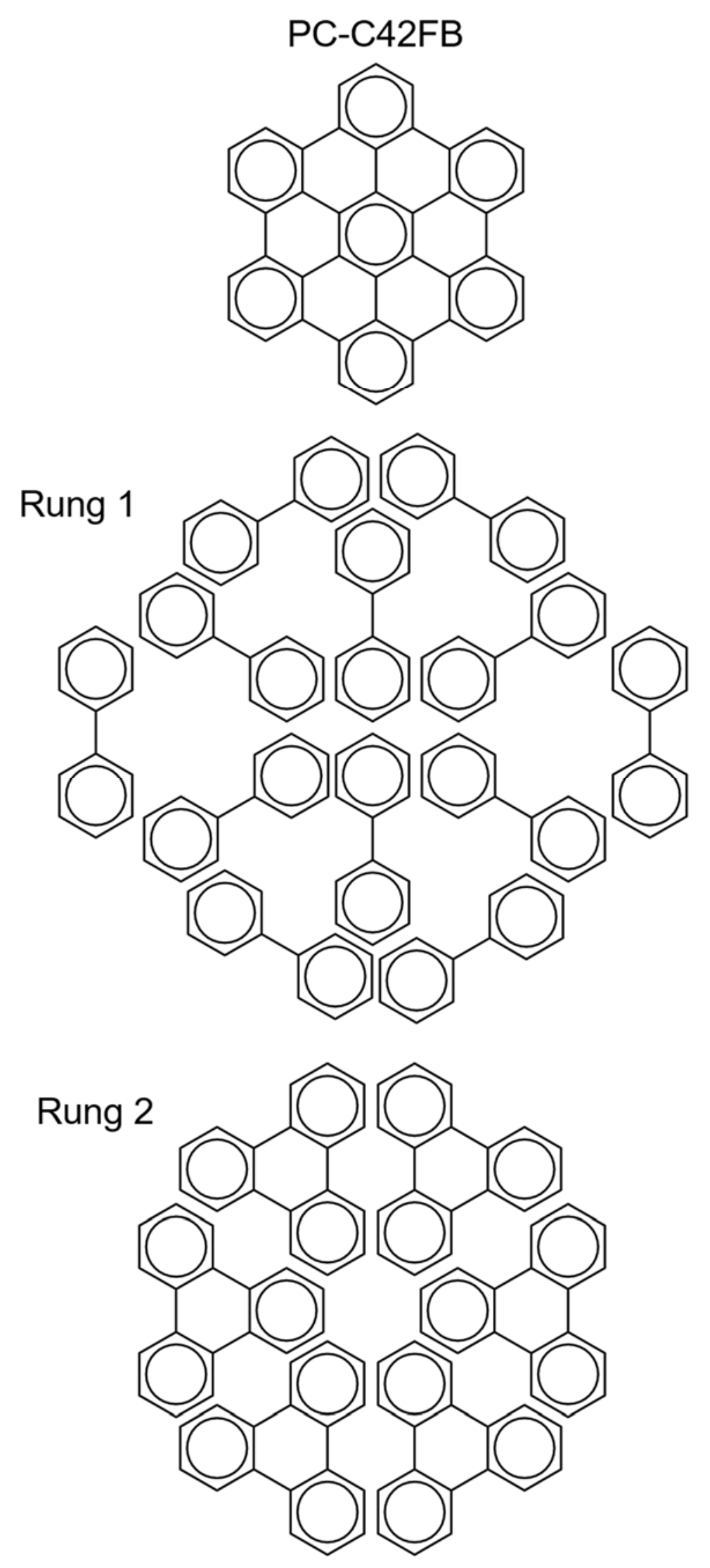




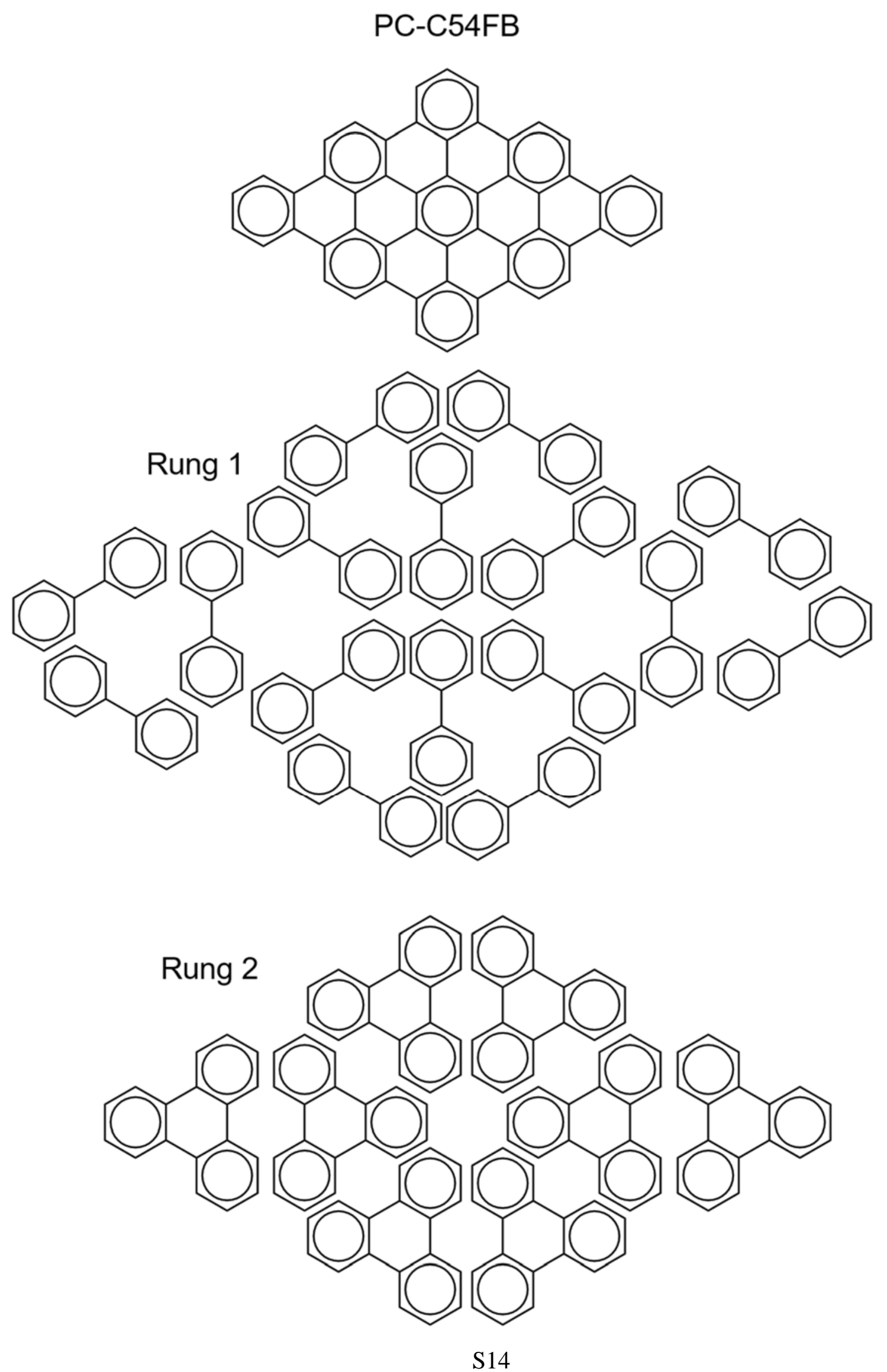




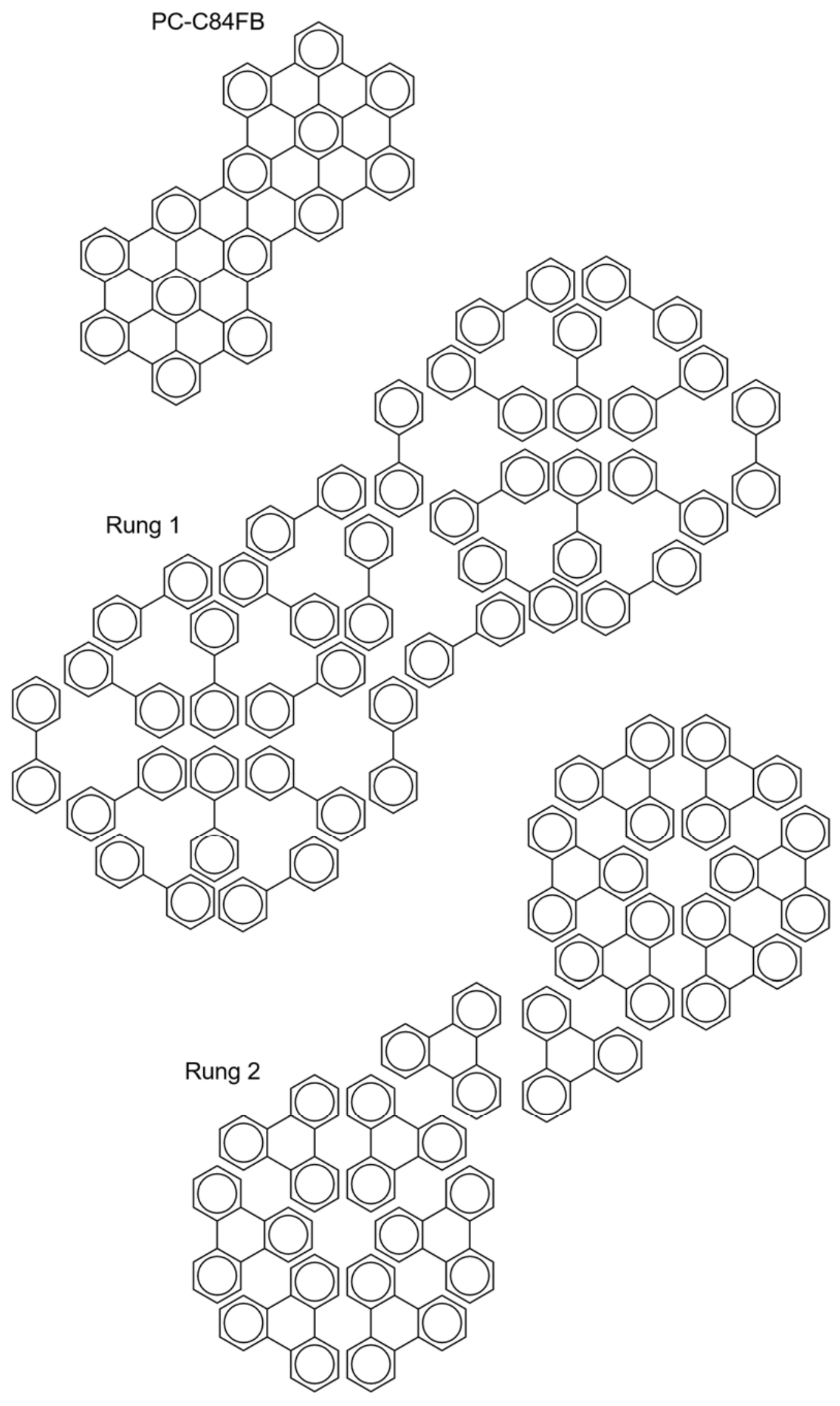

